\title{
Positional voting systems generated by cumulative standings functions
}

\author{
Bonifacio Llamazares · Teresa Peña
}

\begin{abstract}
Positional voting systems are a class of voting systems where voters rank order the candidates from best to worst and a set of winners is selected using the positions of the candidates in the voters' preference orders. Although scoring rules are the best known positional voting systems, this class includes other voting systems proposed in the literature as, for example, the Majoritarian Compromise or the $q$ Approval Fallback Bargaining. In this paper we show that some of these positional voting systems can be integrated in a model based on cumulative standings functions. The proposed model allows us to establish a general framework for the analysis of these voting systems, to extend to them some results in the literature for the particular case of the scoring rules, and also facilitates the study of the social choice properties considered in the paper: monotonicity, Pareto-optimality, immunity to the absolute winner paradox, Condorcet consistency, immunity to the absolute loser paradox and immunity to the Condorcet loser paradox.
\end{abstract}

Keywords Positional voting systems - Cumulative standings functions - Social choice properties

\section{Introduction}

In many voting systems, voters' preferences on a set of candidates are represented by linear orderings (complete, transitive and antisymmetric binary relations). A wellknown procedure to aggregate the preferences of the voters and to obtain the winning candidates consists in assigning a fixed number of points, $s_{k}$, to the $k$-th most preferred candidate of each voter and electing the candidate with the highest score (scoring rules). Besides the scoring rules, there exist other methods (which are less

Bonifacio Llamazares, Teresa Peña

Departamento de Economía Aplicada, Instituto de Matemáticas (IMUVA), Universidad de Valladolid,

Avda. Valle de Esgueva 6, 47011 Valladolid, Spain.

Tel.: +34983186544

Fax: +34983423299

E-mail: boni@eco.uva.es, maitepe@eco.uva.es 
known than the previous ones) that provide a set of winning candidates from the number of first, second, .., $m$ th ranks obtained by them. These procedures are called positional voting systems $(P V S s)^{1}$. Some examples of these voting systems are the Lexicographic rule, the Condorcet's practical method described by Nurmi (1999, p. 21), the Majoritarian Compromise introduced by Sertel and Yilmaz (1999), the q-Approval Fallback Bargaining suggested by Brams and Kilgour (2001) and some models based on mathematical programming techniques proposed by Obata and Ishii (2003), Contreras et al (2005) and Llamazares and Peña (2013) 2 .

It is known that all these methods may provide different sets of winning candidates (in this respect, and for some of them, see Merlin et al (2006), and also Sheikhmohammady et al (2010), where a practical application can be found). So, in order to identify the best rule to use in each situation we need to know which social choice properties are met by each of them. Monotonicity, Pareto-optimality, immunity to the absolute winner paradox, Condorcet consistency, immunity to the absolute loser paradox and immunity to the Condorcet loser paradox are considered as "especially desirable" social choice properties by some authors (see, for instance, Felsenthal (2012)).

In this paper we set up a model that allows us to unify the study of the PVSs above mentioned. In this model we consider for each candidate the number of votes obtained by him/her until each $k$ th place, called his/her cumulative standings (on this, see Fishburn 1974, Moulin 1988, cap. 9, Stein et al 1994, Green et al 1996 and Llamazares and Peña 2009, 2013, 2014), and assign a score to each candidate by using a monotonic (nondecreasing) function of his/her cumulative standings (called cumulative standings function). Those candidates having the highest total score are the winners.

The model proposed allows us to extend to all PVSs generated by cumulative standings functions some known results for the particular case of the scoring rules. We also show that the PVSs previously mentioned can be represented by using this general framework and that new PVSs can be defined by considering other cumulative standings function. Finally, we take advantage of this representation for analyzing their social choice properties.

The paper is organized as follows. In Section 2 we introduce the cumulative standings functions, that derive a score for each candidate from the number of cumulative standings that he/she has obtained. In Section 3 we describe with the help of these functions some of the PVSs proposed in the literature and define a new PVS by using a cumulative standings function equivalent (in this context) to the geometric mean. In Section 4 the cumulative standing functions are used to analyze which desirable social choice properties are met by each of the procedures. Finally, Section 5 is devoted to conclusions. All proofs are in the Appendix.

\footnotetext{
1 As far as we know this term first appears in Gärdenfors (1973).

2 It is worth noting that there exist other PVSs based on mathematical programming techniques that have an important drawback from the point of view of social choice theory: The relative order between two candidates may be altered when the number of first, second, .., $m$ th ranks obtained by other candidates changes, although there are not any variations in the number of first, second, $\ldots, m$ th ranks obtained by both candidates (see Llamazares and Peña 2009). The PVSs with this drawback are not considered in this paper.
} 


\section{PVSs and cumulative standings functions}

Throughout the paper, we will use the following notation: Given two vectors $\mathbf{x}, \mathbf{y} \in$ $\mathbb{R}^{k}, \mathbf{x} \geq \mathbf{y}$ denotes $x_{i} \geq y_{i}$ for all $i \in\{1, \ldots, k\} ; \mathbf{x}>\mathbf{y}$ means that $\mathbf{x} \geq \mathbf{y}$ and $\mathbf{x} \neq \mathbf{y}$; $\mathbf{x} \gg \mathbf{y}$ means that $x_{i}>y_{i}$ for all $i \in\{1, \ldots, k\}$. Moreover, given $a \in \mathbb{R},\lfloor a\rfloor$ will denote the integer part of $a$, i.e., the largest integer smaller than or equal to $a$.

Let $\mathscr{A}=\left\{A_{1}, \ldots, A_{m}\right\}$ be a set of candidates, with $m \geq 3$. A linear order on $\mathscr{A}$ is a complete, transitive and antisymmetric binary relation on $\mathscr{A}$. A linear order on $\mathscr{A}$ where $A_{1}$ is the most preferred candidate, $A_{2}$ is the second most preferred candidate and so on will be written in the usual way as $A_{1} A_{2} \ldots A_{m-1} A_{m}$. Let $\mathscr{P}$ be the set of linear orders on $\mathscr{A}$. A profile $\mathbf{p}$ is a vector of linear orders on $\mathscr{A}$, where each component represents the preferences of a voter. A voting system (also called social choice rule) is a function $C: \bigcup_{n \geq 2} \mathscr{P}^{n} \longrightarrow 2^{\mathscr{A}} \backslash\{\varnothing\}$.

PVSs are a class of voting systems where the set of winners is determined using the positions of the candidates in the voter's preference order, that is, given a profile p, PVSs only need the number of votes obtained by each candidate in each $k$ th place for selecting the winning candidates. In this paper, instead of using those values, we consider the number of votes obtained by each candidate until the $k$ th place (called his/her cumulative standings). In this way, if we denote by $v_{k}^{i}$ the number of $k$ th place ranks that candidate $A_{i}$ occupies, his/her cumulative standings ${ }^{3}, V_{k}^{i}$, will be

$$
V_{k}^{i}=\sum_{l=1}^{k} v_{l}^{i}, \quad k=1, \ldots, m
$$

Notice that if $n$ is the number of voters of a profile $\mathbf{p}$, then $\sum_{i=1}^{m} v_{k}^{i}=n$ for all $k \in\{1, \ldots, m\}$. Therefore,

$$
\sum_{i=1}^{m} V_{k}^{i}=\sum_{i=1}^{m} \sum_{l=1}^{k} v_{l}^{i}=\sum_{l=1}^{k} \sum_{i=1}^{m} v_{l}^{i}=\sum_{l=1}^{k} n=k n .
$$

Once obtained the cumulative standings of each candidate, we assign a score to each of them by using a monotonic function $F$ of his/her cumulative standings. Since the number of cumulative standings in the last place is always the number of voters of the profile $\mathbf{p}$ (that is, $V_{m}^{i}=n$ for all $i \in\{1, \ldots, m\}$ ), this function, called cumulative standings function $(C S F)$, will be defined without considering $V_{m}^{i}$ as variable ${ }^{4}$, i.e.,

$$
F: \mathscr{V} \longrightarrow \mathbb{R}
$$

where

$$
\mathscr{V}=\left\{\left(V_{1}, \ldots, V_{m-1}\right) \in \mathbb{N}^{m-1} \mid 0 \leq V_{1} \leq \cdots \leq V_{m-1}\right\}
$$

\footnotetext{
3 Although the cumulative standings of each candidate depend on the profile $\mathbf{p}$, in order to avoid cumbersome notation we shall omit $\mathbf{p}$ in the notation of these values when there is no possible confusion. When it will be necessary, we will use the notation $V_{k}^{i}$ for the profile $\mathbf{p}, V_{k}^{\prime i}$ for the profile $\mathbf{p}^{\prime}$ and so on.

4 Nevertheless, for convenience we continue to use the notation $V_{m}^{i}$ instead of $n$ when appropriate.
} 
Thus, if $\mathbf{V}^{i}=\left(V_{1}^{i}, V_{2}^{i}, \ldots, V_{m-1}^{i}\right)$ represents the candidate $A_{i}$ 's cumulative standings, the score obtained by candidate $A_{i}$ is $F\left(\mathbf{V}^{i}\right)$ and the candidates can be ordered in the usual way:

$$
A_{i} \succ A_{j} \Leftrightarrow F\left(\mathbf{V}^{i}\right)>F\left(\mathbf{V}^{j}\right) .
$$

Given a CSF $F$, the PVS obtained by the procedure described above will be called the PVS generated by $F^{5}$. Notice that different CSFs can yield the same PVS. In this case we will say that the CSFs are equivalent.

We would like to highlight that the monotonicity property imposed on these functions can be considered a reasonable condition: if $\mathbf{V}^{i} \geq \mathbf{V}^{j}$ (that is, if all the cumulative standings of candidate $A_{i}$ are higher than or equal to those of candidate $A_{j}$ ), it seems natural from a positional perspective that $A_{i} \succcurlyeq A_{j}$, or equivalently,

$$
\mathbf{V}^{i} \geq \mathbf{V}^{j} \Rightarrow F\left(\mathbf{V}^{i}\right) \geq F\left(\mathbf{V}^{j}\right)
$$

To finish this section, it is worth pointing out that the use of CSFs allows us to extend to the field of PVSs some results known in the literature. For example, that the winning candidates with all scoring rules can be easily characterized by means of cumulative standings (see Fishburn 1974 and Stein et al 1994). Given that the CSFs are monotonic, the extension of this result is straightforward.

Theorem 1 Given a profile $\mathbf{p}$, a candidate $A_{i}$ is a winner with all PVSs generated by CSFs if and only if $\mathbf{V}^{i} \geq \mathbf{V}^{j}$ for all $j \neq i$.

\section{Examples of PVSs generated by CSFs}

In this section we recall some PVSs proposed in the literature and show that these systems can be obtained through $\mathrm{CSFs}^{6}$. It is worth noting that, in some cases, the PVSs suggested in the literature only provide a set of winning candidates. However, the use of CSFs determine a weak order among the candidates so that the candidates ordered in first place are the winning candidates given by the PVSs.

We can classify the PVSs analyzed in this paper in the following manner:

1. Classical PVSs, such as the scoring rules and the Lexicographic rule.

2. PVSs based on the trade-off between the quality and quantity of support behind candidates, such as the Condorcet's practical method, the $q$-Approval Fallback Bargaining and the Majoritarian Compromise ${ }^{7}$.

3. PVSs based on scoring rules with variable scoring vectors, such as the models proposed by Obata and Ishii (2003) and Contreras et al (2005).

4. PVSs based on means, such as the Geometric rule.

\footnotetext{
5 Taking into account that the score of each candidate only depends on the number of first, second, ..., $m$ th ranks obtained by him/her, the use of CSFs excludes the PVSs that have the drawback mentioned in the introduction.

6 It is easy to check that the functions considered in this section are monotonic and, therefore, are CSFs.

7 Notice that, although the majoritarian compromise historically precedes $q$-approval fallback bargaining, we first show this PVS for the sake of simplicity of the CSF.
} 


\subsection{Scoring rules}

The scoring rule associated with $\mathbf{s}$ is the social choice rule defined through the scoring vector $\mathbf{s}=\left(s_{1}, \ldots, s_{m}\right) \in \mathbb{R}^{m}$, with $s_{1} \geq s_{2} \geq \cdots \geq s_{m}$ and $s_{1}>s_{m}$, where, for each profile $\mathbf{p}, s_{k}$ points are assigned to the $k$ th most preferred candidate of each voter and the winning candidates are those who receive the highest total number of points. So, the score obtained by candidate $A_{i}$ is $\sum_{k=1}^{m} s_{k} v_{k}^{i}$, where $v_{k}^{i}$ is the number of $k$ th place ranks that candidate $A_{i}$ occupies.

By using the cumulative standings $V_{k}^{i}$, Llamazares and Peña (2014) have shown that scoring rules can be defined through the CSFs

$$
F_{\mathbf{S}}\left(V_{1}, \ldots, V_{m-1}\right)=S_{1} V_{1}+\cdots+S_{m-1} V_{m-1},
$$

where the scoring vectors $\mathbf{S}$ belong to the following set:

$$
\mathscr{S}=\left\{\mathbf{S} \in \mathbb{R}^{m-1} \mid \mathbf{S}>\mathbf{0}, \sum_{k=1}^{m-1} S_{k}=1\right\} .
$$

Concretely, the best-known scoring rules can be represented by means of $F_{\mathbf{S}}$ as follows:

1. Borda rule: A candidate receives no points for being ranked last, one point for being ranked next to last, up to $m-1$ points for being ranked first. In the set of scoring vectors $\mathscr{S}$, Borda rule is obtained when $S_{k}=1 /(m-1)$ for all $k \in$ $\{1, \ldots, m-1\}$; that is,

$$
F_{\mathbf{S}}\left(V_{1}, \ldots, V_{m-1}\right)=\frac{V_{1}+\cdots+V_{m-1}}{m-1} .
$$

So, the score obtained by each candidate is the arithmetic mean of his/her cumulative standings.

2. $k$-approval voting: The candidates with the largest number of votes in the $k$ first positions win $^{8}$. In the set of scoring vectors $\mathscr{S}, k$-approval voting is obtained when $S_{k}=1$ and $S_{i}=0$ for all $i \neq k$; that is,

$$
F_{\mathbf{S}}\left(V_{1}, \ldots, V_{m-1}\right)=V_{k}
$$

It is important to emphasize that $k$-approval voting systems allow us to characterize the winning candidates with all PVSs generated by CSFs. Notice that, given a profile $\mathbf{p}$, a candidate $A_{i}$ is a winning candidate with all $k$-approval voting systems if and only if $\mathbf{V}^{i} \geq \mathbf{V}^{j}$ for all $j \neq i$. So, from Theorem 1 , it is immediate to deduce the following corollary ${ }^{9}$.

Corollary 1 Given a profile $\mathbf{p}$, a candidate $A_{i}$ is a winner with all PVSs generated by CSFs if and only if he/she is a winning candidate with all $k$-approval voting systems.

\footnotetext{
8 The best known special cases of $k$-approval voting are plurality $(k=1)$ and antiplurality $(k=m-1)$

9 This corollary extends to all PVSs generated by CSFs the result obtained for the scoring rules by Saari $(1992,1994)$ (see also Merlin et al 2000).
} 


\subsection{Lexicographic rule}

The lexicographic rule can be seen as a way of breaking ties in the plurality rule. So, in the lexicographic voting rule, the candidate with the highest number of first ranks wins. When several candidates satisfy this condition, the candidate with the highest number of second ranks is chosen from among them and so on.

A CSF of this rule can be obtained by similarity with the lexicographic order of natural numbers: Given two natural numbers with the same number of digits, the natural number with the highest first digit is greater, if the two numbers have the same first digit, the number with the highest second digit is greater and so on. This lexicographic order of natural numbers comes from their representation in base 10, where digits range from 0 to 9 and each position has a value ten times that of the position to its right. For this reason, a unit in a position has a value greater than nine units in all positions to its right. For instance,

$$
1 \cdot 10^{2}+0 \cdot 10^{1}+0 \cdot 10^{0}=100>099=0 \cdot 10^{2}+9 \cdot 10^{1}+9 \cdot 10^{0} .
$$

Taking into account that similar comments can be done for any base- $k$ numerical system and given that $V_{i}$ ranges from 0 to $n$ for all $i \in\{1, \ldots, m-1\}$, the winning can-

didates with the lexicographic rule can be obtained by considering that $V_{1}, \ldots, V_{m-1}$ are the digits of a number represented in the base- $(n+1)$ numerical system and calculating its value, that is, the following CSF:

$$
F_{L}\left(V_{1}, \ldots, V_{m-1}\right)=V_{1}(n+1)^{m-2}+\cdots+V_{m-2}(n+1)+V_{m-1} .
$$

\subsection{Condorcet's practical method}

Under this rule, the candidate with an absolute majority of first ranks wins. If there is no such candidate, the winning candidates are those with the biggest sum of first and second ranks (see, for instance, Nurmi 1999, p. 21).

It is easy to check that the winning candidates can be obtained with the following CSF:

$$
F_{C}\left(V_{1}, \ldots, V_{m-1}\right)=H\left(V_{1}-(\lfloor n / 2\rfloor+1)\right)+\frac{V_{2}}{n},
$$

where $\lfloor x\rfloor$ is the integer part of $x$, i.e., the largest integer smaller than or equal to $x$, and $H$ is the Heaviside step function with $H(0)=1$ (or, equivalently, the characteristic function of the interval $[0, \infty)$ ); that is,

$$
H(x)=\left\{\begin{array}{l}
1, \text { if } x \geq 0 \\
0, \text { if } x<0
\end{array}\right.
$$

The winning candidates with this method can also be obtained with the following equivalent CSF:

$$
F_{C}^{\prime}\left(V_{1}, \ldots, V_{m-1}\right)=\left\lfloor\frac{V_{1}}{\lfloor n / 2\rfloor+1}\right\rfloor+\frac{V_{2}}{n} .
$$




\section{4 q-Approval Fallback Bargaining}

Brams and Kilgour (2001) proposed a procedure where the winning candidates are selected in the following manner: Given $q \in\{1, \ldots, n\}$, if a candidate is ranked first by at least $q$ voters, that candidate wins; otherwise, if this threshold is achieved by some candidate when the first and second ranks are considered, that candidate is chosen; if not, then lower rankings must be added until some candidate achieves the threshold established. Notice that when $q=n$, the procedure is denominated by the authors as Fallback Bargaining and it is also know as the Maximin rule or the Kant-Rawls social compromise (see Hurwicz and Sertel 1999) and it has been characterized by Congar and Merlin (2012).

The winning candidates provided by this method can be obtained by using the following CSF:

$$
F_{q}\left(V_{1}, \ldots, V_{m-1}\right)=H\left(V_{1}-q\right)+\cdots+H\left(V_{m-1}-q\right),
$$

where $H$ is the Heaviside step function with $H(0)=1$. Note that, since $V_{1} \leq \cdots \leq$ $V_{m-1}$, when a summand of this function is equal to 1 , the following summands will also be 1 ; that is, if $H\left(V_{k}-q\right)=1$, then $H\left(V_{l}-q\right)=1$ for all $l \in\{k+1, \ldots, m-1\}$. Therefore, $F_{q}$ returns the value $m-k$, where $k$ is the smaller integer for which $V_{k} \geq q$.

It is worth noting that when $n$ is odd and $q=(n+1) / 2$, the previous CSF determines a weak order among the candidates which coincides with that obtained by a procedure proposed by Bassett and Persky (1999): the Median Voting Rule (see also Gehrlein and Lepelley 2003).

On the other hand, the function $H\left(V_{i}-q\right)$ takes the same values as $\left\lfloor V_{i} / q\right\rfloor$ when $q>n / 2$. Therefore, in this case, we can also use the following CSF to obtain the set of winning candidates chosen by $q$-approval fallback bargaining:

$$
F_{q}^{\prime}\left(V_{1}, \ldots, V_{m-1}\right)=\left\lfloor\frac{V_{1}}{q}\right\rfloor+\cdots+\left\lfloor\frac{V_{m-1}}{q}\right\rfloor .
$$

\subsection{The Majoritarian Compromise}

Sertel and Yilmaz (1999) suggested a model similar to the previous one with $q=$ $\lfloor(n+1) / 2\rfloor$, but when the previous procedure is applied to the $k$ first positions and various winning candidates are obtained, Sertel and Yilmaz proposed to select those with the biggest sum of first, second, ..., $k$ th ranks.

The winning candidates in this model can be obtained with the following CSF:

$$
\begin{aligned}
F_{M}\left(V_{1}, \ldots, V_{m-1}\right)= & \frac{V_{1}}{n} H\left(V_{1}-q\right) \\
& +\left(\frac{V_{2}}{n}+\left(1-\frac{V_{2}}{n}\right) H\left(V_{1}-q\right)\right) H\left(V_{2}-q\right)+\cdots \\
& +\left(\frac{V_{m-1}}{n}+\left(1-\frac{V_{m-1}}{n}\right) H\left(V_{m-2}-q\right)\right) H\left(V_{m-1}-q\right),
\end{aligned}
$$


where $H$ is the Heaviside step function with $H(0)=1$. So, when $V_{1} \geq q$ for various candidates, the first summand is $V_{1} / n$ and the remaining $m-2$ summands are equal to 1 for these candidates. Therefore the candidates with the highest number of first positions win. Analogously, when $V_{1}<q$ for all candidates but $V_{2} \geq q$ for various candidates, the first summand is 0 , the second summand is $V_{2} / n$ and the remaining $m-3$ summands are equal to 1 for these candidates. Therefore the candidates with the highest number of first and second positions win. And so on.

\subsection{Obata and Ishii's method}

One of the most important issues in the context of scoring rules is the choice of the scoring vector, since the winning candidates can vary according to the scores used. To avoid this problem, Obata and Ishii (2003), following the idea of Cook and Kress (1990), proposed to assess each candidate with the most favorable scoring vector for $\mathrm{him} /$ her using a DEA/AR model. In a particular case of this model (when the $L_{1}$ norm is used and the discrimination intensity functions are zero), the score achieved by each candidate is

$$
\begin{gathered}
\widehat{Z}_{i}=\max \sum_{k=1}^{m} s_{k} v_{k}^{i} \\
\text { s.t. } \mathbf{s} \in \widehat{\mathscr{S}}
\end{gathered}
$$

where

$$
\begin{array}{r}
\widehat{\mathscr{S}}=\left\{\mathbf{s} \in \mathbb{R}^{m} \mid \mathbf{s}>\mathbf{0}, \sum_{k=1}^{m} s_{k}=1, s_{k} \geq s_{k+1}(k=1, \ldots, m-1),\right. \\
\left.\sum_{k=1}^{m} s_{k} v_{k}^{j} \leq \sum_{k=1}^{m} s_{k} v_{k}^{i}(\text { for all } j \neq i)\right\} .
\end{array}
$$

The winning candidates ${ }^{10}$ with this model have been characterized by Foroughi et al (2005) and Llamazares and Peña (2009): A candidate $A_{i}$ is a winner if and only if

$$
\max _{k=1, \ldots, m} \frac{V_{k}^{i}}{k} \geq \max _{k=1, \ldots, m} \frac{V_{k}^{j}}{k}
$$

for all $j \in\{1, \ldots, m\}$. Moreover, in this case,

$$
\widehat{Z}_{i}=\max _{k=1, \ldots, m} \frac{V_{k}^{i}}{k}
$$

Therefore, the winning candidates can be obtained using the following CSF:

$$
F_{O I}\left(V_{1}, \ldots, V_{m-1}\right)=\max \left\{V_{1}, \frac{V_{2}}{2}, \ldots, \frac{V_{m-1}}{m-1}, \frac{V_{m}}{m}\right\} .
$$

Notice that, since $V_{k} / k=\left(v_{1}+\cdots+v_{k}\right) / k$ is the value returned by the scoring rule with the normalized scoring vector $\mathbf{s}=(1 / k, \ldots, 1 / k, 0, \ldots, 0)$, the function $F_{O I}$ is equivalent to evaluating the candidates by using the standard normalized $k$-approval voting and choosing the maximum value.

\footnotetext{
10 It is worth noting that the same set of winning candidates can be also obtained through a model proposed by Llamazares and Peña (2013).
} 
3.7 Contreras, Hinojosa and Mármol’s method

As in the previous model, Contreras et al (2005) suggested to assess each candidate with the most favorable scoring vector for him/her by considering several constraints on the scoring vectors. One of the models proposed by these authors is

$$
\begin{aligned}
& \widetilde{Z}_{i}=\max \sum_{k=1}^{m} s_{k} v_{k}^{i} \\
& \text { s.t. } \mathbf{s} \in \widetilde{\mathscr{S}}
\end{aligned}
$$

where

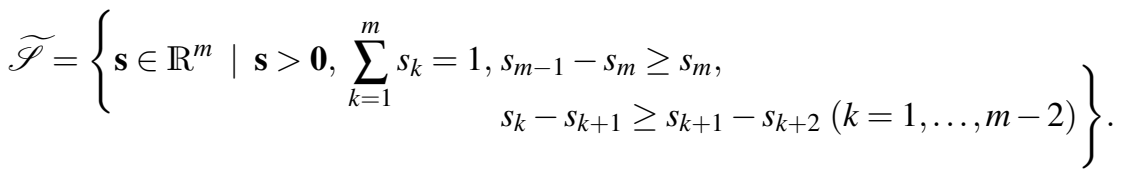

These authors showed that, with this model, the candidate $A_{i}$ 's score is

$$
\widetilde{Z}_{i}=\max _{k=1, \ldots, m}\left\{\sum_{l=1}^{k} \frac{k-l+1}{\frac{k(k+1)}{2}} v_{l}^{i}\right\} .
$$

Therefore, this score also could be obtained using the following CSF:

$$
F_{C H M}\left(V_{1}, \ldots, V_{m-1}\right)=\max \left\{V_{1}, \frac{V_{1}+V_{2}}{3}, \ldots, \frac{2}{m(m+1)} \sum_{k=1}^{m} V_{k}\right\} .
$$

It is worth noting that

$$
\sum_{l=1}^{k} \frac{k-l+1}{\frac{k(k+1)}{2}} v_{l}
$$

is the value returned by the scoring rule associated with the normalized scoring vector

$$
\mathbf{s}=\left(\frac{k}{\frac{k(k+1)}{2}}, \frac{k-1}{\frac{k(k+1)}{2}}, \ldots, \frac{1}{\frac{k(k+1)}{2}}, 0, \ldots, 0\right) .
$$

So, as Contreras et al (2005) have pointed out, the function $F_{C H M}$ is equivalent to evaluating the candidates by using the normalized truncated Borda rules (on this, see Fishburn 1974) and choosing the maximum value.

\subsection{The Geometric rule}

In addition to the PVSs proposed in the literature, others can be established. We have seen above that scoring rules are obtained when weighted means are used as CSFs. For this reason, it would be interesting to know which PVSs result when we use another class of means. For instance, we are going to consider the following CSF, which is equivalent to the geometric mean: 


$$
F_{G}\left(V_{1}, \ldots, V_{m-1}\right)=V_{1} \cdot V_{2} \cdots \cdot V_{m-1} .
$$

It is worth noting that, when this PVS is used, candidates need at least one first position to be winners. This minimum requirement (from a positional point of view) is not fulfilled by some well-known voting systems such as the Borda rule and the Condorcet consistent methods.

\section{Properties of PVSs generated by CSFs}

We next use the cumulative standing functions to analyze which social choice properties are met by each of the procedures described in the previous section ${ }^{11}$. This analysis allows us to compare the different PVSs used in practice from a theoretical point of view.

Concretely, the six properties that we are going to analyze are monotonicity, Pareto-optimality, immunity to the absolute winner paradox, Condorcet-consistency, immunity to the absolute loser paradox and immunity to the Condorcet loser paradox. Monotonicity can be considered as a basic property that must always be satisfied (see, for instance, Fishburn 1982 and Moulin 1988, p. 233). On the other hand, Felsenthal (2012) reviews the main paradoxes afflicting voting procedures and points out the violation of monotonicity or of the last four properties as specially intolerable paradoxes $^{12}$.

It is worth noting that, in some cases, the fulfillment of a property may depend on the number of candidates considered, so our analysis is undertaken for the different values of $m$ (in this regard see the analysis of the Pareto optimality and the absolute loser paradox for the Condorcet's practical method).

With regard to the PVSs, the $q$-Approval Fallback Bargaining deserves a comment. As in the case of qualified majorities, it does not seem plausible to consider the value of $q$ independently of the number of voters, but rather as a percentage of that number. The most common percentages used are $50 \%$ and $100 \%$. When $50 \%$ is considered, the method is similar to the Majoritarian Compromise, so we assume a percentage of $100 \%$, that is $q=n$ (Fallback Bargaining) ${ }^{13}$. Nevertheless, some of the results presented in this paper apply for any $q$ in the range $1 \leq q \leq n$.

\subsection{Monotonicity}

Although the monotonicity of some of the PVSs presented in the previous section is already known, the use of the cumulative standing functions allows us to extend this result to all PVSs generated by these functions.

\footnotetext{
11 Excepting the scoring rules because the results relative to these voting systems are already known (see Llamazares and Peña 2014).

12 It is worth noting that Condorcet-consistency is a controversial property: Although some authors such as Felsenthal (2012) hold that a Condorcet winner, if one exists, ought always to be elected, others, such as Fishburn (1974), question the importance of this property.

13 In this case the cumulative standing function will be denoted by $F_{n}$ instead of $F_{q}$.
} 
Definition 1 A PVS is monotonic if, for every profile $\mathbf{p}$, when some voters raise a winning candidate in their rankings without changing the orders of the remaining candidates, this candidate continues to be a winner.

Theorem 2 All the PVSs generated by CSFs are monotonic.

\subsection{Pareto-optimality}

Definition 2 Let $\mathbf{p}$ be a profile.

1. A candidate $A_{i}$ dominates $A_{j}$ if all the voters strictly prefer $A_{i}$ to $A_{j}$.

2. A candidate $A_{i}$ is Pareto-optimal if there does not exist another candidate that dominates $A_{i}$.

Definition 3 A PVS is Pareto-optimal if, for every profile $\mathbf{p}$, all the winning candidates are Pareto-optimal.

This definition of Pareto-optimality has been used by Moulin (1988, p. 233) and Nurmi (1999, p. 88), among others. Nevertheless, notice that some authors such as Fishburn (1974) and Felsenthal (2012) analyze the susceptibility of some voting systems to the following paradox (that they call the dominated-winner paradox or Pareto paradox): A candidate which is not Pareto-optimal may be a winner while a candidate that dominates him/her may not be. Since the CSFs are monotonic, all PVSs generated by CSFs are immune to this paradox: Given a profile $\mathbf{p}$, if candidate $A_{i}$ dominates candidate $A_{j}$, then $\mathbf{V}^{i} \geq \mathbf{V}^{j}$. Therefore, $F\left(\mathbf{V}^{i}\right) \geq F\left(\mathbf{V}^{j}\right)$ and, consequently, if $A_{j}$ is a winner, then so is $A_{i}$.

The Pareto-optimality of $q$-Approval Fallback Bargaining and the Majoritarian Compromise has been proven by Brams and Kilgour (2001) and Sertel and Yilmaz (1999), respectively. In the following theorem we establish the Pareto-optimality of other PVSs introduced in Section 3.

Theorem 3 The Lexicographic rule, the Obata and Ishii's method, the Contreras, Hinojosa and Mármol's method and the Geometric rule are Pareto-optimal. Moreover, when $m=3$, the Condorcet's practical method is Pareto-optimal.

When $m \geq 4$, the Condorcet's practical method is not Pareto-optimal. To see this fact, it is sufficient to consider 10 voters and a profile $\mathbf{p}$ where

5 voters: $A_{1} A_{2} A_{3} A_{4} \cdots A_{m}$

5 voters: $A_{3} A_{4} A_{1} A_{2} \cdots A_{m}$.

In Table 1 we show the cumulative standings of the candidates. It is easy to check that $A_{1}, A_{2}, A_{3}$ and $A_{4}$ are the winners. However, $A_{2}$ and $A_{4}$ are not Pareto optimal because they are dominated by $A_{1}$ and $A_{3}$, respectively.

\subsection{Absolute winner paradox}

Definition 4 Let $\mathbf{p}$ be a profile. A candidate $A_{i}$ is the absolute winner if he/she is ranked first by an absolute majority of voters. 
Table 1 Cumulative standings of profile $\mathbf{p}$ (Pareto-optimality)

\begin{tabular}{ccccccc}
\hline Candidate & $V_{1}^{i}$ & $V_{2}^{i}$ & $V_{3}^{i}$ & $V_{4}^{i}$ & $\cdots$ & $V_{m-1}^{i}$ \\
\hline$A_{1}$ & 5 & 5 & 10 & 10 & $\cdots$ & 10 \\
$A_{2}$ & 0 & 5 & 5 & 10 & $\cdots$ & 10 \\
$A_{3}$ & 5 & 5 & 10 & 10 & $\cdots$ & 10 \\
$A_{4}$ & 0 & 5 & 5 & 10 & $\cdots$ & 10 \\
$\cdots$ & $\ldots$ & $\ldots$ & $\cdots$ & $\cdots$ & $\cdots$ & $\cdots$ \\
$A_{m}$ & 0 & 0 & 0 & 0 & $\cdots$ & 0 \\
\hline
\end{tabular}

Table 2 Cumulative standings of profile $\mathbf{p}$ (Absolute winner paradox)

\begin{tabular}{cccccc}
\hline Candidate & $V_{1}^{i}$ & $V_{2}^{i}$ & $V_{3}^{i}$ & $\cdots$ & $V_{m-1}^{i}$ \\
\hline$A_{1}$ & 3 & 3 & 5 & $\cdots$ & 5 \\
$A_{2}$ & 2 & 5 & 5 & $\cdots$ & 5 \\
$A_{3}$ & 0 & 2 & 5 & $\cdots$ & 5 \\
$\cdots$ & $\cdots$ & $\cdots$ & $\cdots$ & $\cdots$ & $\cdots$ \\
$A_{m}$ & 0 & 0 & 0 & $\cdots$ & 0 \\
\hline
\end{tabular}

Definition 5 A PVS is immune to the absolute winner paradox if, for every profile p, the absolute winner, whenever he/she exists, is the only winning candidate.

It is obvious that the Lexicographic rule, the Condorcet's practical method and the Majoritarian Compromise are immune to this paradox. In the sequel we establish the immunity to the absolute winner paradox of the Obata and Ishii's method and the Contreras, Hinojosa and Mármol's method.

Theorem 4 The Obata and Ishii's method and the Contreras, Hinojosa and Mármol's method are immune to the absolute winner paradox.

The following example shows that Fallback Bargaining and the Geometric rule are vulnerable to the absolute winner paradox. Consider 5 voters and a profile $\mathbf{p}$ where

3 voters: $A_{1} A_{2} A_{3} \cdots A_{m}$

2 voters: $A_{2} A_{3} A_{1} \cdots A_{m}$.

In this situation the candidate $A_{1}$ is the absolute winner. Table 2 shows the cumulative standings of the candidates ${ }^{14}$. It is easy to check that $F_{n}\left(\mathbf{V}^{2}\right)=m-2$ and $F_{n}\left(\mathbf{V}^{i}\right)<m-2$ for all $i \neq 2$. On the other hand, $F_{G}\left(\mathbf{V}^{1}\right)=9 \cdot 5^{m-3}, F_{G}\left(\mathbf{V}^{2}\right)=10 \cdot 5^{m-3}$ and $F_{G}\left(\mathbf{V}^{i}\right)=0$ for all $i \in\{3, \ldots, m\}$. So, the winner in both cases is $A_{2}$.

\subsection{Condorcet consistency}

Definition 6 Let $\mathbf{p}$ be a profile. A candidate $A_{i}$ is the Condorcet winner if he/she beats all others in pairwise comparison.

Definition 7 A PVS is Condorcet consistent if, for every profile $\mathbf{p}$, the Condorcet winner, whenever he/she exists, is the only winning candidate.

\footnotetext{
${ }^{14}$ Notice that, when $m=3$, the third column of Table 2 is $V_{2}^{i}$ and not $V_{m-1}^{i}$.
} 
Table 3 Cumulative standings of profile $\mathbf{p}$ (Condorcet consistency)

\begin{tabular}{ccccccc}
\hline Candidate & $V_{1}^{i}$ & $V_{2}^{i}$ & $V_{3}^{i}$ & $V_{4}^{i}$ & $\cdots$ & $V_{m-1}^{i}$ \\
\hline$A_{1}$ & 4 & 8 & 10 & 10 & $\cdots$ & 10 \\
$A_{2}$ & 5 & 9 & 11 & 11 & $\cdots$ & 11 \\
$A_{3}$ & 2 & 5 & 11 & 11 & $\cdots$ & 11 \\
$A_{4}$ & 0 & 0 & 1 & 11 & $\cdots$ & 11 \\
$\cdots$ & $\cdots$ & $\cdots$ & $\cdots$ & $\cdots$ & $\cdots$ & $\cdots$ \\
$A_{m}$ & 0 & 0 & 0 & 0 & $\cdots$ & 1 \\
\hline
\end{tabular}

Taking into account that an absolute winner is always a Condorcet winner, a procedure which is vulnerable to the absolute winner paradox is not Condorcet consistent. So, Fallback Bargaining and the Geometric rule are not Condorcet consistent.

On the other hand, the use of cumulative standings functions simplifies the analysis of this property for many PVSs. To see this, we introduce the following definition.

Definition 8 A cumulative standings function $F$ is weakly monotonic when, for every profile $\mathbf{p}, \mathbf{V}^{i} \gg \mathbf{V}^{j}$ implies $F\left(\mathbf{V}^{i}\right)>F\left(\mathbf{V}^{j}\right)$.

It is easy to check that all the scoring rules, the Lexicographic rule, the Condorcet's practical method, the Contreras, Hinojosa and Mármol's method and the Geometric rule are generated by weakly monotonic CSFs.

PVSs generated by weakly monotonic CSFs are not Condorcet consistent. To see this fact, it is sufficient to consider 11 voters and a profile $\mathbf{p}$ where
4 voters: $A_{1} A_{2} A_{3} A_{4} \cdots A_{m}$
2 voters: $A_{2} A_{3} A_{1} A_{4} \cdots A_{m}$
2 voters: $A_{2} A_{1} A_{3} A_{4} \cdots A_{m}$
2 voters: $A_{3} A_{1} A_{2} A_{4} \cdots A_{m}$
1 voter: $A_{2} A_{3} A_{4} A_{5} \cdots A_{1}$.

In Table 3 we show the cumulative standings of the candidates ${ }^{15}$. As we can see, $\mathbf{V}^{2} \gg \mathbf{V}^{1}$. Therefore, if the PVS is generated by a weakly monotonic function, then $A_{1}$ is not a winning candidate although being the Condorcet winner.

The above profile $\mathbf{p}$ can also be used to show that Fallback Bargaining (when $m \geq$ 4), the Majoritarian Compromise and the Obata and Ishii's method are not Condorcet consistent. Since

$$
\begin{array}{lll}
F_{n}\left(\mathbf{V}^{1}\right)=0, & F_{M}\left(\mathbf{V}^{1}\right)=\frac{8}{11}+m-3, & \\
F_{n}\left(\mathbf{V}^{2}\right)=m-3, & F_{M}\left(\mathbf{V}^{2}\right)=\frac{9}{11}+m-3, & \left.F^{1}\right)=4,
\end{array}
$$

$A_{1}$ is not a winning candidate in the PVSs mentioned previously. For the case $m=3$, the following profile allows us to show that Fallback Bargaining is not Concorcet consistent $^{16}$ :

\footnotetext{
15 It is worth noting that, when $m=3$, the third column of Table 3 is $V_{2}^{i}$ and not $V_{m-1}^{i}$.

16 It is worth noting that Brams and Kilgour (2001) and Sertel and Yilmaz (1999) use examples with four and five candidates, respectively, to show that their methods are not Condorcet consistent. Our examples
} 
3 voters: $A_{1} A_{2} A_{3}$

2 voters: $A_{2} A_{3} A_{1}$.

$A_{1}$ is the Condorcet winner although he/she is not a winning candidate.

\subsection{Absolute loser paradox}

Definition 9 Let $\mathbf{p}$ be a profile. A candidate $A_{i}$ is the absolute loser if he/she is ranked last by an absolute majority of voters.

Definition 10 A PVS is immune to the absolute loser paradox if, for every profile $\mathbf{p}$, the absolute loser, whenever he/she exists, is not a winning candidate.

The immunity of the Majoritarian Compromise to the absolute loser paradox has been noted by Sertel and Yilmaz (1999). This property is called by these authors strong no imposition power.

In the following theorem, we establish the vulnerability to this paradox of the remaining PVSs analyzed in this paper.

Theorem 5 The Lexicographic rule, Fallback Bargaining, the Obata and Ishii's method, the Contreras, Hinojosa and Mármol's method and the Geometric rule are vulnerable to the absolute loser paradox. Moreover, the Condorcet's practical method is vulnerable to this paradox when $m>4$ and immune when $m \leq 4$.

\subsection{Condorcet loser paradox}

Definition 11 Let $\mathbf{p}$ be a profile. A candidate $A_{i}$ is the Condorcet loser if an absolute majority of voters prefer each of the remaining candidates to $A_{i}$.

Definition 12 A PVS is immune to the Condorcet loser paradox if, for every profile $\mathbf{p}$, the absolute loser, whenever he/she exists, is not a winning candidate.

Taking into account that an absolute loser is always a Condorcet loser, a procedure which is vulnerable to the absolute loser paradox is also vulnerable to the Condorcet loser paradox. So, the Lexicographic rule, the Condorcet's practical method (with $m>4$ ), Fallback Bargaining, the Obata and Ishii's method, the Contreras, Hinojosa and Mármol's method and the Geometric rule suffer from the last paradox. On the other hand, Sertel and Yilmaz (1999) have shown that the Majoritarian Compromise is vulnerable to the Condorcet loser paradox when $m=5$.

In the sequel, we show that the Condorcet's practical method is vulnerable to the Condorcet loser paradox when $m \leq 4$ and that the Majoritarian Compromise suffers from this paradox independently of the number of the candidates.

First, consider 15 voters, 4 candidates and a profile $\mathbf{p}$ where

show this result independently of the number of the candidates. In this respect, for $m \geq 3$, the no-Condorcet consistency of the Condorcet's practical method and the Majoritarian Compromise has been noted by Merlin et al (2006) using the geometry of voting. 
Table 4 Cumulative standings of profile $\mathbf{p}$ (Condorcet loser paradox, $m=4$ )

\begin{tabular}{cccc}
\hline Candidate & $V_{1}^{i}$ & $V_{2}^{i}$ & $V_{3}^{i}$ \\
\hline$A_{1}$ & 0 & 9 & 11 \\
$A_{2}$ & 6 & 8 & 9 \\
$A_{3}$ & 6 & 8 & 10 \\
$A_{4}$ & 3 & 5 & 15 \\
\hline
\end{tabular}

Table 5 Cumulative standings of profile $\mathbf{p}^{\prime}$ (Condorcet loser paradox, $m=3$ )

\begin{tabular}{ccc}
\hline Candidate & $V_{1}^{\prime i}$ & $V_{2}^{\prime i}$ \\
\hline$A_{1}$ & 3 & 11 \\
$A_{2}$ & 6 & 9 \\
$A_{3}$ & 6 & 10 \\
\hline
\end{tabular}

4 voters: $A_{2} A_{1} A_{4} A_{3}$

2 voters: $A_{2} A_{3} A_{4} A_{1}$

2 voters: $A_{3} A_{1} A_{4} A_{2}$

2 voters: $A_{3} A_{2} A_{4} A_{1}$

2 voters: $A_{3} A_{4} A_{1} A_{2}$

2 voters: $A_{4} A_{1} A_{3} A_{2}$

1 voter: $A_{4} A_{1} A_{2} A_{3}$

It is easy to check that $A_{1}$ is the Condorcet loser. In Table 4 we show the cumulative standings of the candidates. From this table we obtain that $F_{C}\left(\mathbf{V}^{1}\right)=9 / 15$, $F_{C}\left(\mathbf{V}^{2}\right)=F_{C}\left(\mathbf{V}^{3}\right)=8 / 15$ and $F_{C}\left(\mathbf{V}^{4}\right)=5 / 15$. On the other hand, $F_{M}\left(\mathbf{V}^{1}\right)=9 / 15+$ $1, F_{M}\left(\mathbf{V}^{2}\right)=F_{M}\left(\mathbf{V}^{3}\right)=8 / 15+1$ and $F_{M}\left(\mathbf{V}^{4}\right)=15 / 15$. So, the winner with both methods is $A_{1}$.

Now, let us remove candidate $A_{4}$ from the previous profile, maintaining the order of the three remaining candidates. In this way, we obtain a new profile $\mathbf{p}^{\prime}$ with only three candidates where $A_{1}$ is still the Condorcet loser. Table 5 shows the new cumulative standings of the candidates. In this case, $F_{C}\left(\mathbf{V}^{\prime 1}\right)=F_{M}\left(\mathbf{V}^{\prime 1}\right)=11 / 15$, $F_{C}\left(\mathbf{V}^{\prime 2}\right)=F_{M}\left(\mathbf{V}^{\prime 2}\right)=9 / 15$ and $F_{C}\left(\mathbf{V}^{\prime 3}\right)=F_{M}\left(\mathbf{V}^{\prime 3}\right)=10 / 15$. Again, the Condorcet loser is the winner with both methods.

Finally, to show that the Majoritarian Compromise suffers from this paradox for all $m \geq 5$ we use the following example. On one hand, we consider the forward cyclic list of orders ${ }^{17}$ generated by $A_{2} A_{3} \cdots A_{m}$ and place candidate $A_{1}$ in the second position. On the other hand, we consider the forward cyclic list of orders generated by $A_{2} A_{3} \cdots A_{m-1}$ and place candidates $A_{m}$ and $A_{1}$ in the penultimate and last position, respectively. In this way, we have the following profile $\mathbf{p}$ of $2 m-3$ voters:

\footnotetext{
17 According to Fishburn (1974), the forward cyclic list of orders generated by $A_{1} A_{2} \cdots A_{m}$ is the $m$-tuple of orders $\left(A_{1} A_{2} \cdots A_{m-1} A_{m}, A_{2} A_{3} \cdots A_{m} A_{1}, A_{3} A_{4} \cdots A_{1} A_{2}, \ldots, A_{m} A_{1} \cdots A_{m-2} A_{m-1}\right)$.
} 
Table 6 Cumulative standings of profile $\mathbf{p}$ (Condorcet loser paradox, $m \geq 5$ )

\begin{tabular}{ccccc}
\hline Candidate & $V_{1}^{i}$ & $V_{2}^{i}$ & $\cdots$ & $V_{m-1}^{i}$ \\
\hline$A_{1}$ & 0 & $m-1$ & $\cdots$ & $m-1$ \\
$A_{2}$ & 2 & 3 & $\cdots$ & $2 m-4$ \\
$A_{3}$ & 2 & 3 & $\cdots$ & $2 m-4$ \\
$\cdots$ & $\cdots$ & $\cdots$ & $\cdots$ & $\cdots$ \\
$A_{m-1}$ & 2 & 3 & $\cdots$ & $2 m-4$ \\
$A_{m}$ & 1 & 1 & $\cdots$ & $2 m-4$ \\
\hline
\end{tabular}

1 voter: $A_{2} A_{1} A_{3} \cdots A_{m-1} A_{m}$

1 voter: $A_{3} A_{1} A_{4} \cdots A_{m} A_{2}$

1 voter: $A_{4} A_{1} A_{5} \cdots A_{2} A_{3}$

voter: $A_{m} A_{1} A_{2} \cdot A_{m-2} A_{m-1}$

1 voter: $A_{2} A_{3} \cdots A_{m-1} A_{m} A_{1}$

1 voter: $A_{3} A_{4} \cdots A_{2} A_{m} A_{1}$

1 voter: $A_{4} A_{5} \cdots A_{3} A_{m} A_{1}$

1 voter: $A_{m-1} A_{2} \cdots A_{m-2} A_{m} A_{1}$

It is easy to check that $A_{1}$ is the Condorcet loser. In Table 6 we show the cumulative standings of the candidates according to the profile $\mathbf{p}$.

For $m \geq 5$ none of the candidates have a majority of first ranks. In this situation we have

$$
\begin{aligned}
& F_{M}\left(\mathbf{V}^{1}\right)=\frac{m-1}{2 m-3}+m-3, \\
& F_{M}\left(\mathbf{V}^{i}\right) \leq \frac{3}{2 m-3}+m-3 \quad(\text { for all } i \in\{2, \ldots, m\}),
\end{aligned}
$$

that is, $F_{M}\left(\mathbf{V}^{1}\right)>F_{M}\left(\mathbf{V}^{i}\right)$ for all $i \in\{2, \ldots, m\}$. Therefore, $A_{1}$ is the winner.

\section{Conclusion}

In this paper we have established a general framework based on cumulative standings functions for the analysis of some positional voting systems. The proposed model has allowed us to extend some results appeared in the literature. For example, we have extended to all PVSs generated by CSFs the characterization of the winning candidates under every scoring rule given by Fishburn (1974). Also, we have shown that a candidate is a winner with all PVSs generated by CSFs if and only if he/she is the winning candidate with all $k$-approval systems, $k=1, \ldots, m-1$. Thus, we extend to all PVSs generated by CSFs the result obtained by Saari $(1992,1994)$, (see also Merlin et al 2000), for scoring rules. On the other hand, the proposed model has facilitated the analysis of these voting systems through various properties that can be established for cumulative standings functions. For example, given that cumulative standing functions are monotonic, it is straightforward to check that all PVSs 
Table 7 Social choice properties of some PVSs

\begin{tabular}{|c|c|c|c|c|c|c|}
\hline & M & $\mathrm{PO}$ & IAWP & $\mathrm{CC}$ & IALP & ICLP \\
\hline Lexicographic rule & $\checkmark$ & $\checkmark$ & $\checkmark$ & & & \\
\hline Condorcet's practical method & $\checkmark$ & $\sqrt{ }(m=3)$ & $\checkmark$ & & $\sqrt{ }(m \leq 4)$ & \\
\hline Fallback Bargaining & $\checkmark$ & $\checkmark$ & & & & \\
\hline Majoritarian Compromise & $\checkmark$ & $\checkmark$ & $\checkmark$ & & $\checkmark$ & \\
\hline Obata and Ishii's method & $\checkmark$ & $\checkmark$ & $\checkmark$ & & & \\
\hline Contreras, Hinojosa and Mármol's method & $\checkmark$ & $\checkmark$ & $\checkmark$ & & & \\
\hline Geometric rule & $\checkmark$ & $\checkmark$ & & & & \\
\hline
\end{tabular}

M: Monotonicity, PO: Pareto-optimality, IAWP: Immunity to the absolute winner paradox, CC: Condorcet consistency, IALP: Immunity to the absolute loser paradox, ICLP: Immunity to the Condorcet loser paradox.

generated by these function are monotonic. Also, if the CSF that generate a PVS is weakly monotonic, it is easy to prove that this PVS is not Condorcet consistent. Furthermore, the use of cumulative standings together with the representation of PVSs by means of CSFs facilitates the analysis of other properties of the PVSs such as Pareto-optimality, the immunity to the absolute winner paradox, the immunity to the absolute loser paradox and the immunity to the Condorcet loser paradox. Given that in some cases the satisfaction of a property may depend on the number of candidates considered, our analysis of the axioms listed above has been undertaken for different values of this variable. Table 7 summarizes the results obtained in this respect.

Acknowledgements The authors are very grateful to two anonymous referees for valuable suggestions, comments and references. This work is partially supported by the Spanish Ministry of Economy and Competitiveness (Projects ECO2011-24200 and ECO2012-32178) and the Junta de Castilla y León (Consejería de Educación, Project VA066U13).

\section{A Appendix}

Proof of Theorem 2. Let $F$ be a CSF, let $\mathbf{p}$ be a profile and let $A_{i}$ be a winning candidate for this profile. Suppose that some voters raise $A_{i}$ in their rankings without changing the orders of the remaining candidates. Let $\mathbf{p}^{\prime}$ be this new profile. It is straightforward to check that $\mathbf{V}^{\prime i} \geq \mathbf{V}^{i}$ and $\mathbf{V}^{\prime j} \leq \mathbf{V}^{j}$ for all $j \neq i$. Since $A_{i}$ is a winning candidate and $F$ is monotonic we have

$$
F\left(\mathbf{V}^{\prime i}\right) \geq F\left(\mathbf{V}^{i}\right) \geq F\left(\mathbf{V}^{j}\right) \geq F\left(\mathbf{V}^{\prime j}\right)
$$

for all $j \neq i$. Therefore, $A_{i}$ continues to be a winning candidate for the new profile $\mathbf{p}^{\prime}$.

Before proving Theorem 3, we give the following lemma.

Lemma 1 If the candidate $A_{i}$ is not Pareto-optimal, then $V_{1}^{i}=0$ and there exists another candidate $A_{j}$ with $V_{k}^{i} \leq V_{k-1}^{j}$ for all $k \in\{2, \ldots, m\}$. 
Proof. If $A_{i}$ is not Pareto-optimal, there exists another candidate $A_{j}$ that is preferred to $A_{i}$ by all voters, so $V_{1}^{i}=0$. On the other hand, when a voter places candidate $A_{i}$ in the $k$ th position, that voter will have ranked $A_{j}$ higher. Therefore, $V_{k}^{i} \leq V_{k-1}^{j}$ for all $k \in\{2, \ldots, m\}$.

Proof of Theorem 3. In all cases we are going to prove that, given a profile $\mathbf{p}$, if a candidate $A_{i}$ is not Pareto-optimal, then $A_{i}$ cannot be a winning candidate.

1. Lexicographic rule: If $A_{i}$ is not Pareto-optimal, then, by Lemma $1, V_{1}^{i}=0$ and, consequently, $A_{i}$ is not a winning candidate.

2. Condorcet's practical method: Consider $m=3$. If $A_{i}$ is not Pareto-optimal, then he/she is dominated by a candidate $A_{j}$. We distinguish two cases:

(a) If $V_{1}^{j}>\lfloor n / 2\rfloor$, then $A_{j}$ is the only winner.

(b) If $V_{1}^{j} \leq\lfloor n / 2\rfloor$, then, by Lemma $1, V_{1}^{i}=0, V_{2}^{j} \geq V_{3}^{i}=n$ and $V_{2}^{i} \leq V_{1}^{j}<n$. So, $F_{C}\left(\mathbf{V}^{j}\right)=1$ and $F_{C}\left(\mathbf{V}^{i}\right)<1$. Therefore, $A_{i}$ cannot be a winning candidate

3. Obata and Ishii's method: If $A_{i}$ is not Pareto-optimal, then, by Lemma $1, V_{1}^{i}=0$ and there exists another candidate $A_{j}$ with $V_{k}^{i} \leq V_{k-1}^{j}$ for all $k \in\{2, \ldots, m\}$. Let $l \in\{2, \ldots, m\}$ such that

$$
F_{O I}\left(\mathbf{V}^{i}\right)=\max \left\{V_{1}^{i}, \frac{V_{2}^{i}}{2}, \ldots, \frac{V_{m-1}^{i}}{m-1}, \frac{V_{m}^{i}}{m}\right\}=\frac{V_{l}^{i}}{l} .
$$

Since $V_{l}^{i}>0$ and $V_{l}^{i} \leq V_{l-1}^{j}$, we have

$$
F_{O I}\left(\mathbf{V}^{i}\right)=\frac{V_{l}^{i}}{l}<\frac{V_{l-1}^{j}}{l-1} \leq F_{O I}\left(\mathbf{V}^{j}\right) .
$$

Therefore, $A_{i}$ is not a winning candidate.

4. Contreras, Hinojosa and Mármol's method: If $A_{i}$ is not Pareto-optimal, then, by Lemma $1, V_{1}^{i}=0$ and there exists another candidate $A_{j}$ with $V_{k}^{i} \leq V_{k-1}^{j}$ for all $k \in\{2, \ldots, m\}$. Let $l \in\{2, \ldots, m\}$ such that

$$
F_{C H M}\left(\mathbf{V}^{i}\right)=\max \left\{V_{1}^{i}, \frac{V_{1}^{i}+V_{2}^{i}}{3}, \ldots, \frac{2}{m(m+1)} \sum_{k=1}^{m} V_{k}^{i}\right\}=\frac{2}{l(l+1)} \sum_{k=1}^{l} V_{k}^{i}
$$

Since $\sum_{k=1}^{l} V_{k}^{i}>0$ and $\sum_{k=1}^{l} V_{k}^{i} \leq \sum_{k=1}^{l-1} V_{k}^{j}$, we have

$$
F_{C H M}\left(\mathbf{V}^{i}\right)=\frac{2}{l(l+1)} \sum_{k=1}^{l} V_{k}^{i}<\frac{2}{(l-1) l} \sum_{k=1}^{l-1} V_{k}^{j} \leq F_{C H M}\left(\mathbf{V}^{j}\right) .
$$

Therefore, $A_{i}$ is not a winning candidate.

5. Geometric rule: If $A_{i}$ is not Pareto-optimal, then, by Lemma $1, V_{1}^{i}=0$ and, consequently, $F_{G}\left(\mathbf{V}^{i}\right)=0$. So, $A_{i}$ is not a winning candidate.

Proof of Theorem 4. 
1. Obata and Ishii's method: Let $A_{i}$ be the absolute winner of a profile $\mathbf{p}$. In this case, $V_{1}^{i} \geq\lfloor n / 2\rfloor+1$; so, $F_{O I}\left(\mathbf{V}^{i}\right) \geq\lfloor n / 2\rfloor+1$. Now, consider $j \neq i$. Then $V_{k}^{j} / k \leq n / 2$ for all $k \in\{1, \ldots, m\}$. Therefore, $F_{O I}\left(\mathbf{V}^{j}\right) \leq n / 2<\lfloor n / 2\rfloor+1 \leq F_{O I}\left(\mathbf{V}^{i}\right)$, for all $j \neq i$. Consequently, $A_{i}$ is the only winner.

2. Contreras, Hinojosa and Mármol's method: Let $A_{i}$ be the absolute winner of a profile $\mathbf{p}$. In this case, $V_{1}^{i} \geq\lfloor n / 2\rfloor+1$; so, $F_{C H M}\left(\mathbf{V}^{i}\right) \geq\lfloor n / 2\rfloor+1$. Now, consider $j \neq i$. Note that

$$
F_{C H M}\left(\mathbf{V}^{j}\right)=\max _{l=1, \ldots, m}\left\{\frac{2}{l(l+1)} \sum_{k=1}^{l} V_{k}^{j}\right\}
$$

and that $V_{1}^{j} \leq n-(\lfloor n / 2\rfloor+1)$. Then, for all $l \in\{1, \ldots, m\}$, we have

$$
\begin{aligned}
\frac{2}{l(l+1)} \sum_{k=1}^{l} V_{k}^{j} & \leq \frac{2}{l(l+1)}(n-(\lfloor n / 2\rfloor+1)+(l-1) n) \\
& =\frac{2}{l(l+1)}(l n-(\lfloor n / 2\rfloor+1))=2\left(\frac{n}{l+1}-\frac{\lfloor n / 2\rfloor+1}{l(l+1)}\right) .
\end{aligned}
$$

We are going to prove that the maximum value of the last expression is achieved when $l=2$. We distinguish two cases:

(a) If $l=1$, then

$$
\frac{n}{2}-\frac{\lfloor n / 2\rfloor+1}{2} \leq \frac{n}{3}-\frac{\lfloor n / 2\rfloor+1}{6} \Leftrightarrow \frac{n}{6} \leq \frac{\lfloor n / 2\rfloor+1}{3} \Leftrightarrow n \leq 2(\lfloor n / 2\rfloor+1),
$$

which is true.

(b) If $l \in\{3, \ldots, m\}$, then

$$
\begin{aligned}
\frac{n}{l+1}-\frac{\lfloor n / 2\rfloor+1}{l(l+1)} & \leq \frac{n}{3}-\frac{\lfloor n / 2\rfloor+1}{6} \\
& \Leftrightarrow(\lfloor n / 2\rfloor+1) \frac{l(l+1)-6}{6 l(l+1)} \leq n \frac{l-2}{3(l+1)} \\
& \Leftrightarrow(\lfloor n / 2\rfloor+1) \frac{(l+3)(l-2)}{l} \leq 2 n(l-2) \\
& \Leftrightarrow(\lfloor n / 2\rfloor+1)(l+3) \leq 2 l n,
\end{aligned}
$$

which is true.

Therefore,

$$
\begin{aligned}
F_{C H M}\left(\mathbf{V}^{j}\right) & \leq 2\left(\frac{n}{3}-\frac{\lfloor n / 2\rfloor+1}{6}\right) \\
& <2\left(\frac{2(\lfloor n / 2\rfloor+1)}{3}-\frac{\lfloor n / 2\rfloor+1}{6}\right)=\lfloor n / 2\rfloor+1 \leq F_{C H M}\left(\mathbf{V}^{i}\right),
\end{aligned}
$$

for all $j \neq i$. Consequently, $A_{i}$ is the only winner. 
Table 8 Cumulative standings of profile $\mathbf{p}$ (Absolute loser paradox)

\begin{tabular}{ccccccc}
\hline Candidate & $V_{1}^{i}$ & $V_{2}^{i}$ & $V_{3}^{i}$ & $\cdots$ & $V_{m-2}^{i}$ & $V_{m-1}^{i}$ \\
\hline$A_{1}$ & $4 m-5$ & $4 m-5$ & $4 m-5$ & $\cdots$ & $4 m-5$ & $4 m-5$ \\
$A_{2}$ & 4 & 11 & 19 & $\cdots$ & $4(2 m-5)-1$ & $4(2 m-3)-1$ \\
$A_{3}$ & 4 & 12 & 19 & $\cdots$ & $4(2 m-5)-1$ & $4(2 m-3)-1$ \\
$\cdots$ & $\cdots$ & $\cdots$ & $\cdots$ & $\cdots$ & $\cdots$ & $\cdots$ \\
$A_{m-1}$ & 4 & 12 & 20 & $\cdots$ & $4(2 m-5)$ & $4(2 m-3)-1$ \\
$A_{m}$ & 4 & 12 & 20 & $\cdots$ & $4(2 m-5)$ & $4(2 m-3)$ \\
\hline
\end{tabular}

Proof of Theorem 5. First, we are going to prove that the Lexicographic rule, the Condorcet's practical method (with $m>4$ ), Fallback Bargaining, the Obata and Ishii's method, the Contreras, Hinojosa and Mármol's method and the Geometric rule are vulnerable to the absolute loser paradox. Consider twice the forward cyclic list of orders generated by $A_{2} A_{3} \cdots A_{m}$. In the first forward cyclic list we place candidate $A_{1}$ in the first position and in the second forward cyclic list we place $A_{1}$ in the last position. Now we consider a profile $\mathbf{p}$ of $8 m-9$ voters where each order is considered 4 times but the first one, which is considered 3 times:

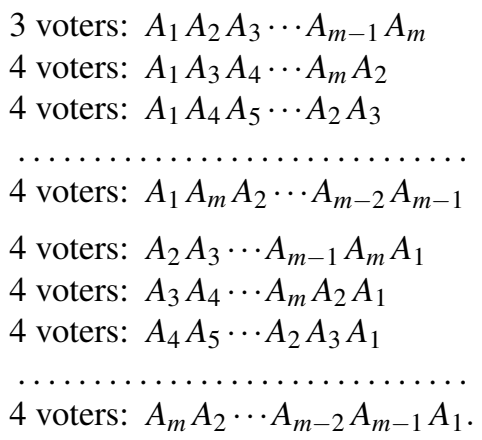

Notice that $A_{1}$ is the absolute loser in the profile p. In Table 8 we show the cumulative standings of the candidates according to this profile. It is worth noting that $V_{k}^{m}=4(2 k-1)$ for all $k \in\{1, \ldots, m-1\}$.

We are going to show that $A_{1}$ is a winning candidate for the PVSs listed in the statement of Theorem 5 and, consequently, these PVSs are vulnerable to the absolute loser paradox. Given that $\mathbf{V}^{m} \geq \mathbf{V}^{i}$ for all $i \in\{2, \ldots, m-1\}$, for any CSF $F$ we have $F\left(\mathbf{V}^{m}\right) \geq F\left(\mathbf{V}^{i}\right)$ (and, therefore, $A_{m} \succcurlyeq A_{i}$ ) for all $i \in\{2, \ldots, m-1\}$. Let us see that $A_{1} \succcurlyeq A_{m}$ for the PVSs considered:

1. Lexicographic rule: Since $4 m-5>4$ for all $m \geq 3$, we have that $A_{1} \succ A_{m}$.

2. Condorcet's practical method: Since neither $A_{1}$ nor $A_{m}$ have a majority of first ranks and $4 m-5>12$ for all $m \geq 5$, we have that $A_{1} \succ A_{m}$.

3. Fallback Bargaining: Since $V_{m-1}^{i}<8 m-9=n$ when $i \in\{1, m\}$, we have that $F_{n}\left(\mathbf{V}^{1}\right)=F_{n}\left(\mathbf{V}^{m}\right)=0$ and, consequently, $A_{1} \succcurlyeq A_{m}$ 
4. Obata and Ishii's method: Since $V_{k}^{m}=4(2 k-1)$ for all $k \in\{1, \ldots, m-1\}$, the scores of candidates $A_{1}$ and $A_{m}$ are

$$
\begin{aligned}
& F_{O I}\left(\mathbf{V}^{1}\right)=\max \left\{4 m-5, \frac{4 m-5}{2}, \ldots, \frac{4 m-5}{m-1}, \frac{8 m-9}{m}\right\}, \\
& F_{O I}\left(\mathbf{V}^{m}\right)=\max \left\{4,6, \ldots, \frac{4(2 m-3)}{m-1}, \frac{8 m-9}{m}\right\} .
\end{aligned}
$$

It is straightforward to check the following inequalities:

$$
\begin{aligned}
4 m-5>\frac{8 m-9}{m} & (\text { for all } m \geq 3), \\
\frac{4(2 m-3)}{m-1}>\frac{8 m-9}{m} & (\text { for all } m \geq 3),
\end{aligned}
$$

and

$$
\frac{V_{k+1}^{m}}{k+1}=\frac{4(2(k+1)-1)}{k+1}>\frac{4(2 k-1)}{k}=\frac{V_{k}^{m}}{k} \quad(\text { for all } k \in\{1, \ldots, m-2\}) .
$$

Therefore, we get

$$
F_{O I}\left(\mathbf{V}^{1}\right)=4 m-5, \quad F_{O I}\left(\mathbf{V}^{m}\right)=\frac{4(2 m-3)}{m-1} .
$$

Given that

$$
4 m-5>\frac{4(2 m-3)}{m-1} \quad(\text { for all } m \geq 3)
$$

we have $A_{1} \succ A_{m}$.

5. Contreras, Hinojosa and Mármol's method: The score obtained by candidates $A_{1}$ is

$$
\begin{aligned}
F_{C H M}\left(\mathbf{V}^{1}\right)=\max \{4 m-5, & \frac{2}{3}(4 m-5), \ldots, \frac{2}{m}(4 m-5), \\
& \left.\frac{2}{m(m+1)}((m-1)(4 m-5)+(8 m-9))\right\} .
\end{aligned}
$$

It is straightforward to check that the inequality

$$
4 m-5>\frac{2}{m(m+1)}((m-1)(4 m-5)+(8 m-9))
$$

is satisfied for all $m \geq 3$. Therefore, $F_{C H M}\left(\mathbf{V}^{1}\right)=4 m-5$.

To calculate the score of candidate $A_{m}$ we take into account that

$$
\sum_{l=1}^{k} V_{l}^{m}=4 \sum_{l=1}^{k}(2 l-1)=4 k^{2}
$$


for all $k \in\{1, \ldots, m-1\}$. Therefore

$$
\begin{aligned}
\frac{2}{k(k+1)} \sum_{l=1}^{k} V_{l}^{m} & =\frac{8 k}{k+1} \quad(\text { for all } k \in\{1, \ldots, m-1\}), \\
\frac{2}{m(m+1)} \sum_{l=1}^{m} V_{l}^{m} & =\frac{2}{m(m+1)}\left(4(m-1)^{2}+(8 m-9)\right) .
\end{aligned}
$$

It is easy to check the following inequalities:

$$
\begin{aligned}
\frac{8 k}{k+1} & <\frac{8(k+1)}{k+2} \quad(\text { for all } k \in\{1, \ldots, m-2\}), \\
\frac{2}{m(m+1)}\left(4(m-1)^{2}+(8 m-9)\right) & <\frac{8(m-1)}{m} .
\end{aligned}
$$

Therefore

$$
F_{C H M}\left(\mathbf{V}^{m}\right)=\frac{8(m-1)}{m} .
$$

Given that

$$
4 m-5>\frac{8(m-1)}{m} \quad(\text { for all } m \geq 3),
$$

we have $A_{1} \succ A_{m}$.

6. Geometric rule: The scores of candidates $A_{1}$ and $A_{m}$ are

$$
\begin{aligned}
& F_{G}\left(\mathbf{V}^{1}\right)=(4 m-5)^{m-1} \\
& F_{G}\left(\mathbf{V}^{m}\right)=4^{m-1} \prod_{k=1}^{m-1}(2 k-1)=\frac{4^{m-1}(2 m-2) !}{2^{m-1}(m-1) !}=2^{m-1} \frac{(2 m-2) !}{(m-1) !} .
\end{aligned}
$$

We are going to prove that, for all $m \geq 3$,

$$
2^{m-1} \frac{(2 m-2) !}{(m-1) !}<(4 m-5)^{m-1} .
$$

The proof is by induction on $m$. If $m=3$, then $48<49$. Suppose now the result is true for $m=k$, and let us see that the claim holds for $m=k+1$. By hypothesis of induction we have

$$
2^{k} \frac{(2 k) !}{k !}=2 \frac{(2 k-1) 2 k}{k} \cdot 2^{k-1} \frac{(2 k-2) !}{(k-1) !}<4(2 k-1)(4 k-5)^{k-1} .
$$

Since

$$
\begin{aligned}
(4 k-1)^{k} & =(4 k-1)((4 k-5)+4)^{k-1} \\
& >(4 k-2)\left((4 k-5)^{k-1}+(k-1) 4(4 k-5)^{k-2}\right) \\
& >(4 k-2)\left((4 k-5)^{k-1}+(4 k-5)^{k-1}\right) \\
& =4(2 k-1)(4 k-5)^{k-1},
\end{aligned}
$$


we get

$$
2^{k} \frac{(2 k) !}{k !}<(4 k-1)^{k}
$$

Therefore $A_{1} \succ A_{m}$.

Next we are going to prove that the Condorcet's practical method is immune to the absolute loser paradox when $m \leq 4$. Let $A_{i}$ be the absolute loser of a profile $\mathbf{p}$. Then $V_{1}^{i}<\lfloor n / 2\rfloor+1$. We are going to prove that there exists $j \neq i$ such that $V_{2}^{j} \geq V_{2}^{i}$ and, consequently, $A_{i}$ cannot be a winning candidate.

Given that $V_{2}^{i} \leq n-(\lfloor n / 2\rfloor+1)=\lfloor(n-1) / 2\rfloor$ and $\sum_{j=1}^{m} V_{2}^{j}=2 n$, then we have $\sum_{j \neq i} V_{2}^{j} \geq 2 n-\lfloor(n-1) / 2\rfloor$. Therefore, there exists $j \neq i$ such that

$$
V_{2}^{j} \geq \frac{2 n-\lfloor(n-1) / 2\rfloor}{m-1}
$$

Since

$$
\frac{2 n-\lfloor(n-1) / 2\rfloor}{m-1}>\lfloor(n-1) / 2\rfloor \Leftrightarrow 2 n>m\lfloor(n-1) / 2\rfloor \Leftrightarrow \frac{2}{m} n>\lfloor(n-1) / 2\rfloor,
$$

which is true when $m \leq 4$, we get

$$
V_{2}^{j} \geq \frac{2 n-\lfloor(n-1) / 2\rfloor}{m-1}>\lfloor(n-1) / 2\rfloor \geq V_{2}^{i} .
$$

\section{References}

Bassett GW, Persky J (1999) Robust voting. Public Choice 99(3-4):299-310

Brams SJ, Kilgour DM (2001) Fallback bargaining. Group Decis Negot 10(4):287-316

Congar R, Merlin V (2012) A characterization of the maximin rule in the context of voting. Theor Decis 72(1):131-147

Contreras I, Hinojosa MA, Mármol AM (2005) A class of flexible weight indices for ranking alternatives. IMA J Manag Math 16(1):71-85

Cook WD, Kress M (1990) A data envelopment model for aggregating preference rankings. Management Sci 36(11):1302-1310

Felsenthal DS (2012) Review of paradoxes afflicting procedures for electing a single candidate. In: Felsenthal DS, Machover M (eds) Electoral Systems: Paradoxes, Assumptions, and Procedures, Studies in Choice and Welfare, Springer, Berlin, chap 3, pp 19-91

Fishburn PC (1974) Paradoxes of voting. Am Polit Sci Rev 68(2):537-546

Fishburn PC (1982) Monotonicity paradoxes in the theory of elections. Discrete Appl Math 4(2):119-134 Foroughi AA, Jones DF, Tamiz M (2005) A selection method for a preferential election. Appl Math Comput 163(1):107-116

Gärdenfors P (1973) Positionalist voting functions. Theor Decis 4(1):1-24

Gehrlein WV, Lepelley D (2003) On some limitations of the median voting rule. Public Choice 117(12):177-190

Green RH, Doyle JR, Cook WD (1996) Preference voting and project ranking using DEA and crossevaluation. Eur J Oper Res 90(3):461-472

Hurwicz L, Sertel MR (1999) Designing mechanisms, in particular for electoral systems: the majoritarian compromise. In: Sertel MR (ed) Economic Design and Behaviour, Macmillan, London

Llamazares B, Peña T (2009) Preference aggregation and DEA: An analysis of the methods proposed to discriminate efficient candidates. Eur J Oper Res 197(2):714-721 
Llamazares B, Peña T (2013) Aggregating preferences rankings with variable weights. Eur J Oper Res 230(2):348-355

Llamazares B, Peña T (2014) Scoring rules and social choice properties: some characterizations. Theor Decis. In press

Merlin V, Tataru M, Valognes F (2000) On the probability that all decision rules select the same winner. J Math Econ 33(2):183-207

Merlin V, Özkal-Sanver I, Sanver MR (2006) Properties of majoritarian compromise, efficient compromise, and related compromise rules. Paper presented in the 8th International Meeting of the Society for Social Choice and Welfare, Istanbul

Moulin H (1988) Axioms of Cooperative Decision Making, Econometric Society Monographs, vol 15. Cambridge University Press, Cambridge

Nurmi H (1999) Voting Paradoxes and How to Deal with Them. Springer-Verlag, Berlin

Obata T, Ishii H (2003) A method for discriminating efficient candidates with ranked voting data. Eur J Oper Res 151(1):233-237

Saari DG (1992) Millions of election outcomes from a single profile. Soc Choice Welfare 9(4):277-306

Saari DG (1994) Geometry of Voting, Studies in Economic Theory, vol 3. Springer-Verlag, Berlin

Sertel MR, Yilmaz B (1999) The majoritarian compromise is majoritarian-optimal and subgame-perfect implementable. Soc Choice Welfare 16(4):615-627

Sheikhmohammady M, Kilgour DM, Hipel KW (2010) Modeling the Caspian Sea negotiations. Group Decis Negot 19(2):149-168

Stein WE, Mizzi PJ, Pfaffenberger RC (1994) A stochastic dominance analysis of ranked voting systems with scoring. Eur J Oper Res 74(1):78-85 